\title{
Analysis of Mutations in Epidermal Growth Factor Receptor Gene in Korean Patients with Non-small Cell Lung Cancer: Summary of a Nationwide Survey
}

Sang Hwa Lee · Wan Seop Kim Yoo Duk Choi ${ }^{1}$ Jeong Wook Seo $^{2}$ Joung Ho Han ${ }^{3} \cdot$ Mi Jin Kim ${ }^{4}$ Lucia Kim ${ }^{5} \cdot$ Geon Kook Lee ${ }^{6}$ Chang Hun Lee $\cdot$ Mee Hye $\mathrm{Oh}^{8}$ Gou Young Kim ${ }^{9}$. Sun Hee Sung ${ }^{10}$ Kyo Young Lee ${ }^{11}$. Sun Hee Chang ${ }^{12}$ Mee Sook Rho ${ }^{13} \cdot$ Han Kyeom Kim ${ }^{14}$ Soon Hee Jung ${ }^{15}$. Se Jin Jang ${ }^{16}$ The Cardiopulmonary Pathology Study Group of Korean Society of Pathologists

Department of Pathology, Konkuk University School of Medicine, Seoul; ' ${ }^{\text {Chonnam National Univer- }}$ sity Medical School, Gwangju; ${ }^{2}$ Seoul National University College of Medicine, Seoul; 3 Samsung Medical Center, Sungkyunkwan University School of Medicine, Seoul; ${ }^{4}$ Yeungnam University College of Medicine, Daegu; ${ }^{5}$ nha University College of Medicine, Incheon; ${ }^{\circledR}$ National Cancer Center, Goyang; 'Busan National University School of Medicine, Busan; ${ }^{8}$ Soonchunhyang University Cheonan Hospital, Soonchunhyang University College of Medicine, Cheonan; ${ }^{K}$ Kyung Hee University School of Medicine, Seoul; ${ }^{10}$ Ewha Womans University School of Medicine, Seoul; ${ }^{11}$ Seoul St. Mary's Hospital, Catholic University of Korea, Seoul; ${ }^{12}$ nje University Ilsan Paik Hospital, Goyang; ${ }^{13}$ Dong-A University College of Medicine, Busan; ${ }^{14}$ Korea University College of Medicine, Seoul; ${ }^{15}$ Yonsei University Wonju College of Medicine, Wonju; ${ }^{16}$ Asan Medical Center, University of Ulsan College of Medicine, Seoul, Korea

Received: April 24, 2015

Revised: September 13, 2015

Accepted: September 14, 2015

\section{Corresponding Author}

Wan Seop Kim, MD, PhD

Department of Pathology, Konkuk University Medical Center, Konkuk University School of Medicine, 120-1 Neungdong-ro, Gwangjin-gu, Seoul 05030, Korea

Tel: +82-2-2030-5642

Fax: +82-2-2030-5629

E-mail: wskim@kuh.ac.kr
Background: Analysis of mutations in the epidermal growth factor receptor gene (EGFR) is important for predicting response to EGFR tyrosine kinase inhibitors. The overall rate of EGFR mutations in Korean patients is variable. To obtain comprehensive data on the status of EGFR mutations in Korean patients with lung cancer, the Cardiopulmonary Pathology Study Group of the Korean Society of Pathologists initiated a nationwide survey. Methods: We obtained 1,753 reports on EGFR mutations in patients with lung cancer from 15 hospitals between January and December 2009. We compared EGFR mutations with patient age, sex, history of smoking, histologic diagnosis, specimen type, procurement site, tumor cell dissection, and laboratory status. Results: The overall EGFR mutation rate was $34.3 \%$ in patients with non-small cell lung cancer (NSCLC) and $43.3 \%$ in patients with adenocarcinoma. EGFR mutation rate was significantly higher in women, never smokers, patients with adenocarcinoma, and patients who had undergone excisional biopsy. EGFR mutation rates did not differ with respect to patient age or procurement site among patients with NSCLC. Conclusions: EGFR mutation rates and statuses were similar to those in published data from other East Asian countries. 
Lung cancer is the leading cause of cancer-related death in Korea, accounting for approximately $20 \%$ of all cancer deaths. ${ }^{1}$ Non-small cell lung cancer (NSCLC) accounts for more than $85 \%$ of all lung cancers, and the majority of patients with NSCLC present at an advanced cancer stage (stage III or IV). ${ }^{2}$ In the last decade, several studies have been performed on the molecular stratification of NSCLC in order to provide targeted treatment based on activating or driver mutations in these tumors. Activating mutations in the epidermal growth factor receptor gene (EGFR) can be used as therapeutic targets for treatment of NSCLC. In the Iressa Pan-Asia Study, tumors with EGFR mutations showed a $71.2 \%$ clinical response to first-line treatment with gefitinib, while tumors with wild-type EGFR showed only a $1.1 \%$ response. ${ }^{3}$ Since then, several randomized control studies have shown an association between activating EGFR mutation and response to EGFR tyrosine kinase inhibitors (TKIs). ${ }^{4-9}$ Patient selection is important for using EGFR TKIs as the first-line treatment. At present, analysis of EGFR mutations is the accepted method for identifying patient response to EGFR TKIs. Direct DNA sequencing is a standard method for identifying mutations and is commonly used in the Asia-Pacific region. ${ }^{10}$ Any routinely available pathological specimen can be used for analyzing EGFR mutations, including formalin-fixed, paraffin-embedded tissues from surgical resections; small tissue biopsies; or cell block preparations.

Several publications have reported the prevalence of EGFR mutations in patients with NSCLC. ${ }^{10-14}$ The rates of EGFR mutations are higher in Asian countries than in Western countries. Further, rates of EGFR mutations in Korean patients range from $17.4 \%$ to $51.3 \% .^{10,15-22}$ Therefore, we performed a nationwide study of EGFR mutations in Korean patients with NSCLC in order to provide reliable information on the incidence and characteristics of EGFR mutations. This study was led by the Korean Cardiopulmonary Pathology Study Group.

\section{MATERIALS AND METHODS}

In all, 1,826 reports of EGFR mutation in patients with lung cancer were collected from 15 hospitals between January and December 2009 (Fig. 1). Of these, 24 reports of patients with small cell carcinoma and 49 reports of patients with malignancies from other than lung primary tumors were excluded from the study. Finally, 1,544 reports of primary tumor and 209 reports of metastatic tumor were included in the study. EGFR mutation status was compared with patient age, sex, history of smoking, histologic diagnosis, specimen type, procurement site,

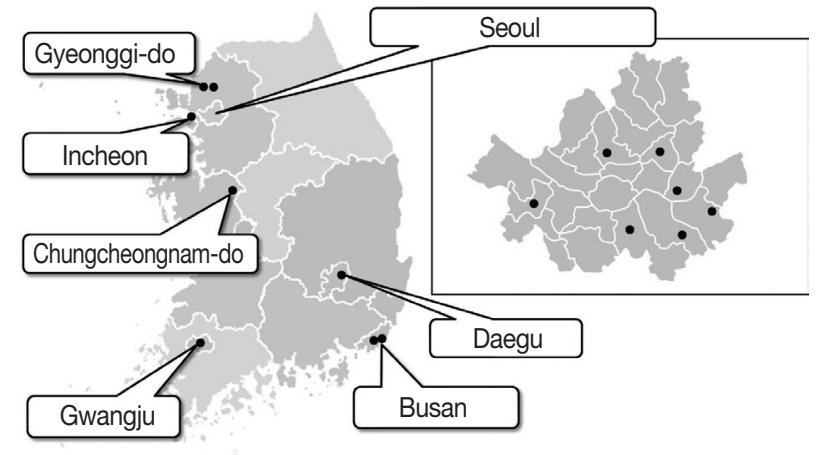

Fig. 1. Geographical distribution of the 15 hospitals in Korea.

tumor cell dissection, and laboratory status. Smoker status was defined as having a greater than 10 pack-year history and currently smoking cigarettes every day or most days. An ex-smoker was someone who had smoked more than 100 cigarettes in their lifetime and who does not currently smoke. A light smoker was defined as a current smoker with a less than 10 pack-year history. A never-smoker was an adult who had never smoked a cigarette or who smoked fewer than 100 cigarettes in their lifetime.

Tumor specimens were divided into three types, namely, biopsy, cytology, and excision specimens. Biopsy specimens included small biopsy specimens obtained by performing bronchoscopic biopsy, transbronchial lung biopsy, percutaneous needle biopsy, pleural biopsy, or needle biopsy of metastatic sites. Cytology included cytologic specimens such as sputum, bronchial washing/ brushing, pleural fluids, and aspiration biopsy cytology of primary or metastatic sites. Excision specimens included specimens obtained by performing excisional surgical biopsy such as segmentectomy, lobectomy, pneumonectomy, and metastasectomy. Procurement sites were divided into two types, namely, metastasis and primary sites. Tumor dissection indicated whether or not to perform microdissection of tumor cells. Laboratory status was classified into two types: in-house mutation testing, indicating that EGFR mutation analysis was performed in the hospital's laboratory facility, and out-sourced mutation testing, indicating that EGFR mutation analysis was not performed in the hospital's laboratory facility. All participants in the study were active members of the Korean Cardiopulmonary Pathology Study Group. This study was approved by the Institutional Review Board of Konkuk University Medical Center (No. KUH 1210011). 


\section{Statistical analysis}

All statistical analyses were performed using SPSS ver. 18.0 (SPSS Inc., Chicago, IL, USA). Chi-square test and Fisher exact test were used to determine the correlations between EGFR mutation status and clinicopathological parameters. A p-value of < .05 was considered statistically significant.

\section{RESULTS}

\section{Patient characteristics}

The average age of 1,753 patients with NSCLC was $62.74 \pm$ 11.31 years (range, 16 to 89 years); of these, 875 patients (49.9\%) were aged $\geq 65$ years. Of the 1,753 patients, $1,000(57 \%)$ were men and 753 (43\%) were women. Of the 1,753 patients, 555 (31.7\%) were smokers, 39 (2.2\%) were light smokers, 170 (9.7\%) were ex-smokers, 849 (48.4\%) were never-smokers, and 140 (8.0\%) patients had an unknown smoking history. Of the specimens used for EGFR mutation testing, 114 (6.5\%) were cytology specimens, 1,066 (60.8\%) were biopsy specimens, and 573 (32.7\%) were excision specimens (Fig. 2). With respect to procurement sites, specimens from 1,544 patients (88.1\%) were procured from primary tumor sites, while those from $209 \mathrm{pa}$ tients (11.9\%) were procured from metastatic sites. In all, 1,012 patients $(57.7 \%)$ underwent tumor microdissection, while the remaining 741 patients (42.3\%) did not. The histological types of the tumor specimens were as follows: adenocarcinoma in 1,292 (73.7\%), squamous cell carcinoma in 347 (19.8\%), NSCLC type undetermined in 69 (3.9\%), pleomorphic carcinoma in $13(0.7 \%)$, large cell neuroendocrine cell carcinoma in 12 $(0.7 \%)$, large cell carcinoma in nine $(0.5 \%)$, sarcomatoid carcinoma in five $(0.3 \%)$, carcinoid tumor in two $(0.1 \%)$, mucoepidermoid carcinoma in two $(0.1 \%)$, carcinosarcoma in one $(0.05 \%)$, and lymphoepithelioma-like carcinoma in one (0.05\%). Among the 1,753 EGFR tests, 1,299 (7 institutions, 74.1\%) were performed within the same pathology laboratory, and 454 (8 insti-

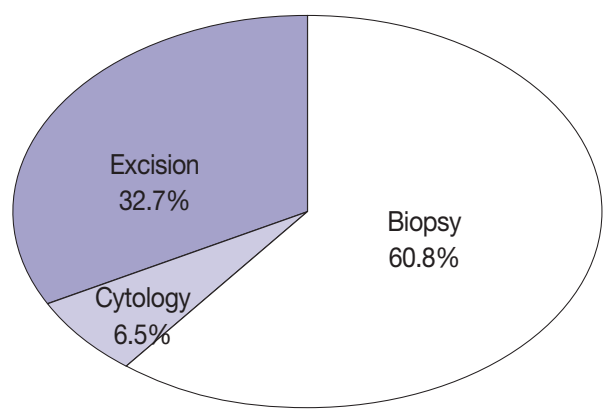

Fig. 2. Specimen types used for EGFR mutation testing. EGFR, epidermal growth factor receptor. tutions, 25.9\%) were performed in outside laboratories. EGFR mutations in specimens obtained from 14 hospitals were identified by direct sequencing, while those in specimens obtained from the remaining hospital were identified by pyrosequencing. Characteristics of patients included in the study are summarized in Table 1.

\section{Frequency of EGFR mutations}

In all, 601 cases of EGFR mutation (34.3\%) were detected in NSCLC. Of these 601 patients, 560 (43.3\%) had adenocarcino$\mathrm{ma}, 30(8.6 \%)$ had squamous cell carcinoma, eight (11.6\%) had NSCLC type undetermined, two (15.4\%) had pleomorphic carcinoma, and one (8.3\%) had large cell neuroendocrine carcino-

Table 1. Characteristics of patients

\begin{tabular}{lc}
\hline Characteristic & No. (\%) \\
\hline Age, mean (range, yr) & $62.7(16-89)$ \\
$<65$ & $878(50.1)$ \\
$\geq 65$ & $875(49.9)$ \\
Sex & \\
Female & $753(43)$ \\
\hline Male & $1,000(57)$ \\
Smoking history & \\
Smoker & $555(31.7)$ \\
Light smoker & $39(2.2)$ \\
Ex-smoker & $170(9.7)$ \\
Never smoker & $849(48.4)$ \\
Unknown & $140(8.0)$ \\
Specimen type & \\
Cytology & $114(6.5)$ \\
Biopsy & $1,066(60.8)$ \\
Excision & $573(32.7)$ \\
Procurement site & \\
Primary & $1,544(88.1)$ \\
Metastasis & $209(11.9)$ \\
Tumor dissection & \\
No & $741(42.3)$ \\
Yes & $1,012(57.7)$ \\
Histologic type & \\
Adenocarcinoma & $1,299(74.1)$ \\
Squamous cell carcinoma & \\
Non-small cell carcinoma & $1,292(73.7)$ \\
Pleomorphic carcinoma & $347(19.8)$ \\
Large cell neuroendocrine carcinoma & $69(3.9)$ \\
Large cell carcinoma & $13(0.7)$ \\
Sarcomatoid carcinoma & $12(0.7)$ \\
Carcinoid tumor & $9(0.5)$ \\
Mucoepidermoid carcinoma & $5(0.3)$ \\
Carcinosarcoma & $2(0.1)$ \\
\hline Lymphoepithelioma-like carcinoma & $2(0.1)$ \\
\hline
\end{tabular}


ma. In all, 389 never-smokers with adenocarcinoma (52.4\%) had EGFR mutations. Of the 601 patients with NSCLC who had EGFR mutations, 30 (5\%), 313 (52.1\%), 31 (5.2\%), and $205(34.1 \%)$ had mutations in exons 18, 19, 20, and 21, respectively; in addition, 22 patients $(3.7 \%)$ had double mutations (Fig. 3). Further, 13 patients (2.2\%) had T790M mutation; of these, four patients had only the T790M mutation.

Interestingly, specimens obtained from two in-house laboratories showed low EGFR mutation rates of $16.7 \%$ (2/12 specimens) and $9.3 \%$ ( $4 / 43$ specimens) in patients with NSCLC, respectively, and $28.6 \%$ (2/7 specimens) and $11.5 \%$ (3/26 specimens) in patients with adenocarcinoma and never-smokers (Fig. 4). In brief, one laboratory that detected a $16.7 \%$ mutation rate in patients with NSCLC analyzed tumor specimens from 12 patients. Of these 12 patients, 10 had adenocarcinoma, one had large cell carcinoma, and one had NSCLC. Of the 10 patients with adenocarcinoma, seven, two, and one were never-smokers,

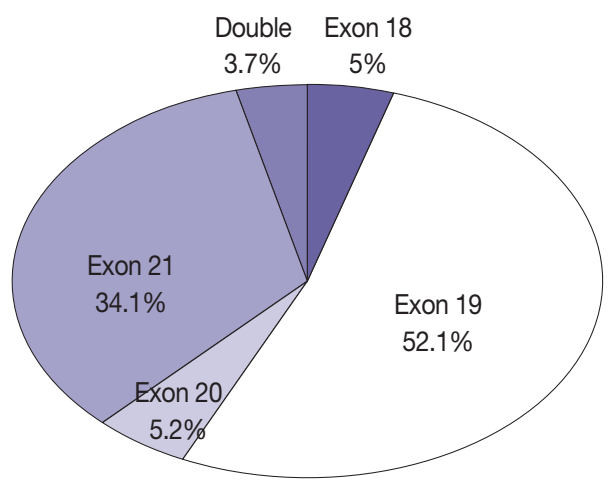

Fig. 3. Frequency of mutations according to exons: 601 mutations in 1,753 specimens from patients with non-small cell lung cancer.

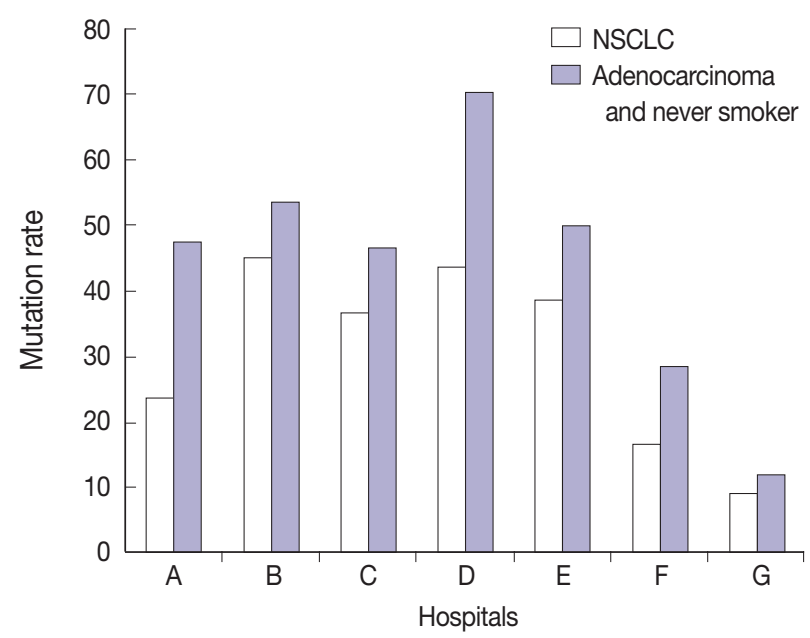

Fig. 4. EGFR mutation rates in in-house laboratories. EGFR, epidermal growth factor receptor. ex-smokers, and smoker, respectively. All the specimens were obtained by surgical excision and were analyzed by tumor microdissection. The laboratory that detected a $9.3 \%$ mutation rate in patients with NSCLC analyzed tumor specimens from 43 patients. Of these 43 patients, 41 had adenocarcinoma, one had NSCLC, and one had squamous cell carcinoma. Of the 41 patients with adenocarcinoma, 25, 6, and 10 were never-smokers, ex-smokers, and smokers, respectively. Of the 43 specimens analyzed in this laboratory, six were surgical excision specimens, 29 were biopsy specimens, and eight were cytology specimens. All 43 specimens were analyzed without tumor microdissection. A total of four EGFR mutations were found, and all of them were adenocarcinoma. They included three never-smokers and one ex-smoker. Moreover, of the four mutations, two were detected in excision specimens and two were detected in biopsy specimens.

A total of 125 EGFR mutations were detected in a total of 430 male patients with adenocarcinoma who had a smoking history.

\section{Differences in EGFR mutation status}

Differences in EGFR mutation status according to clinicopathological variables are summarized in Table 2. Among patients with NSCLC, female $(\mathrm{p}<.001)$, age $<65$ years $(\mathrm{p}=.007)$, light or no smoking $(\mathrm{p}<.001)$, excision specimen $(\mathrm{p}=.002)$, and in-house $E G F R$ mutation testing ( $\mathrm{p}<.001)$ were correlated with significantly higher $E G F R$ mutation rate. Among patients with adenocarcinoma, female $(\mathrm{p}<.001)$, light or no smoking $(\mathrm{p}<.001)$, excision specimen $(\mathrm{p}<.001)$, tumor microdissection $(\mathrm{p}=.001)$, and in-house EGFR mutation testing ( $\mathrm{p}=.046)$ were correlated with significantly higher EGFR mutation rate. According to specimen status, the EGFR mutation rate was $48.1 \%, 47.2 \%$, and $63.4 \%$ in cytology, biopsy, and excision specimen, respectively, from a total of 742 patients with adenocarcinoma and never-smoker status. According to laboratory status, the EGFR mutation rate in patients with NSCLC $(38.2 \%$ vs $23.1 \%, \mathrm{p}<$ .001 ) and adenocarcinoma ( $44.7 \%$ vs $37.6 \%, \mathrm{p}=.046$ ) was significantly higher in in-house tested specimens. Among patients with squamous cell carcinoma, female ( $\mathrm{p}=.001)$ and light to no smoking $(\mathrm{p}=.017)$ were correlated with significantly higher EGFR mutation rate.

\section{DISCUSSION}

The present study identified the frequencies of EGFR mutations in Korean patients with NSCLC. The EGFR mutation rate was $34.3 \%$ and $43.3 \%$ among patients with NSCLC and adeno- 
Table 2. EGFR mutation status according to the clinicopathological variables

\begin{tabular}{|c|c|c|c|c|c|c|c|c|c|}
\hline \multirow{2}{*}{ Variable } & \multicolumn{3}{|c|}{ NSCLC } & \multicolumn{3}{|c|}{ Adenocarcinoma } & \multicolumn{3}{|c|}{ SqCC } \\
\hline & Negative & Positive & $p$-value & Negative & Positive & $p$-value & Negative & Positive & $p$-value \\
\hline Sex & & & $<.001$ & & & $<.001$ & & & .001 \\
\hline Female & $374(49.7)$ & $379(50.3)$ & & $314(46.5)$ & $361(53.5)$ & & 31 (75.6) & $10(24.4)$ & \\
\hline Male & 778 (77.8) & 222 (22.2) & & $418(67.7)$ & 199 (32.3) & & $286(93.5)$ & $20(6.5)$ & \\
\hline Age (yr) & & & .007 & & & .911 & & & .565 \\
\hline$<65$ & $551(62.7)$ & $328(37.3)$ & & $399(56.5)$ & 307 (43.5) & & $100(90.1)$ & $11(9.9)$ & \\
\hline$>65$ & $601(68.8)$ & $273(31.2)$ & & $333(56.8)$ & $253(43.2)$ & & $217(91.9)$ & $19(8.1)$ & \\
\hline Smoking & & & $<.001$ & & & $<.001$ & & & .017 \\
\hline Ex-smoker & $143(84.1)$ & 27 (15.9) & & 88 (77.2) & $26(22.8)$ & & $48(98)$ & $1(2)$ & \\
\hline Light smoker & $22(56.4)$ & $17(43.6)$ & & $17(53.1)$ & $15(46.9)$ & & $2(66.7)$ & 1 (33.3) & \\
\hline Never smoker & $442(52.1)$ & $407(47.9)$ & & $353(47.6)$ & $389(52.4)$ & & $58(82.9)$ & $12(17.1)$ & \\
\hline Smoker & $446(80.4)$ & 109 (19.6) & & $227(70.7)$ & 94 (29.3) & & $168(92.8)$ & $13(7.2)$ & \\
\hline Unknown & $99(70.7)$ & 41 (29.3) & & 47 (56.6) & $36(43.4)$ & & 41 (93.2) & $3(6.8)$ & \\
\hline Specimen type & & & .002 & & & $<.001$ & & & .535 \\
\hline Biopsy & 728 (68.3) & $338(31.7)$ & & $453(59.8)$ & $305(40.2)$ & & $215(90.7)$ & $22(9.3)$ & \\
\hline Cytology & 80 (70.2) & $34(29.8)$ & & 70 (67.3) & 34 (32.6) & & & & \\
\hline Excision & $344(60)$ & $229(40)$ & & $209(48.6)$ & $221(51.4)$ & & $102(92.7)$ & $8(7.3)$ & \\
\hline Procurement site & & & .797 & & & .586 & & & .38 \\
\hline Metastasis & $139(66.5)$ & $70(33.5)$ & & 95 (58.6) & $67(41.4)$ & & 17 (100) & $0(0)$ & \\
\hline Primary & $1,013(65.6)$ & $531(34.4)$ & & $637(56.4)$ & $493(43.6)$ & & $300(90.9)$ & $30(9.1)$ & \\
\hline Tumor dissection & & & .362 & & & .001 & & & .105 \\
\hline No & $478(64.5)$ & $263(35.5)$ & & $401(61.3)$ & $253(38.7)$ & & $45(84.9)$ & 8 (15.1) & \\
\hline Yes & $674(66.6)$ & $338(33.4)$ & & $331(51.9)$ & $307(48.1)$ & & $272(92.5)$ & $22(7.5)$ & \\
\hline Laboratory status & & & $<.001$ & & & .046 & & & .238 \\
\hline In-house & 803 (61.8) & 496 (38.2) & & $581(55.3)$ & $469(44.7)$ & & $144(89.4)$ & 17 (10.6) & \\
\hline Out-sourcing & 349 (76.9) & 105 (23.1) & & $151(62.4)$ & 91 (37.6) & & $173(93)$ & $13(7)$ & \\
\hline
\end{tabular}

Values are presented as number (\%).

EGFR, epidermal growth factor receptor; NSCLC, non-small cell lung cancer; SqCC, squamous cell carcinoma.

carcinoma, respectively, and $52.4 \%$ among never-smokers with adenocarcinoma. The frequencies were in the range of those previously reported in Korean studies, with $17.4 \%-40.8 \%$ in NSCLC, $21.5 \%-54.4 \%$ in adenocarcinoma, and 47\%-64.9\% in adenocarcinoma with never-smokers. ${ }^{10,15-21}$ The results of the present study were also in the range of those from other Asian countries $30 \%-61.1 \%$ in NSCLC and $44.1 \%-67.4 \%$ in adenocarcinoma) and showed a high mutation rate compared with those reported in Western countries (4.5\%-13.3\% in NSCLC and $16 \%$ in adenocarcinoma). ${ }^{3,11,12,23}$ Recently, a large retrospective database study was performed on EGFR mutation testing practices in the Asia-Pacific region. ${ }^{10}$ EGFR mutation rates among patients with NSCLC reported in the present study were very similar to those reported by Yatabe et al. ${ }^{10}$ (39.6\% and $35.8 \%$ EGFR mutation rate among newly diagnosed patients with NSCLC) in the Asia-Pacific region and Korea, respectively, in 2011. The overall distribution pattern of EGFR mutations (i.e., high mutation rates in female patients, never-smokers, and patients with adenocarcinoma) was similar to that reported in previous studies.
The most frequent mutation was an exon 19 deletion, and the most frequent drug resistance-associated mutation was T790M. Chan et al. ${ }^{24}$ identified EGFR mutation hot spots in exons 19 (48\%) and 21 (41\%) in 3,023 specimens. The highest incidence of mutations in EGFR was observed for L858R, del(E746A750), and del(E749-T751), in that order. Shi et al. ${ }^{25}$ reported $43 \%$ and $42.6 \%$ mutation rates in exons 19 and 21 , respectively, among 1,450 specimens, with the most common drug resistance-associated mutation being S768I. The present study reported $52.1 \%$ and $34.1 \%$ mutation rates in exons 19 and 21 , respectively. However, the sum of mutation rates in exons 19 and 21 was $86.2 \%$, which was similar to that reported in studies performed in other countries.

Two laboratories in the present study detected low EGFR mutation rates. Commonalities between these two institutions included a period less than 1 year after starting the EGFR mutation analysis and use of the direct sequencing method. Wellequipped laboratories and technicians skilled at performing EGFR mutation analysis, active engagement of pathologists in molecular testing, and quality assurance were important for ob- 
taining accurate results.

A high incidence of EGFR mutations (29.7\%) has been reported in Korean male smokers with adenocarcinoma. ${ }^{20}$ In the present study, EGFR mutation rate was $29.1 \%$ in male patients with adenocarcinoma who had a smoking history (125 out 430 patients). This result supports the recommendation of a previous study that EGFR mutation tests should be performed in all patients with adenocarcinoma regardless of sex or smoking history. ${ }^{20}$

Because minimally invasive diagnostic procedures are often used in the diagnostic workup of lung cancer, small tissue specimens and, more importantly, cytology specimens might be the only specimens available for EGFR mutation analysis. In the present study, a total of $73.3 \%$ of specimens were cytology or biopsy samples. However, these specimens showed a significantly low mutation rate compared to excision specimens in patients with NSCLC even in patients with adenocarcinoma and neversmokers. Although our results showed significantly higher mutation rates in surgically resected specimens, many studies have reported that small biopsy and cytology specimens are more suitable for performing mutation testing. ${ }^{26-29}$

A quick and accurate test for detecting EGFR mutations is very important for proper selection of patients for EGFR TKI therapy. This highlights the need for standard guidelines specific to medical conditions in Korea for EGFR mutation testing. Many methods are available for detecting EGFR mutations, and these methods have different advantages and disadvantages. However, there is no consensus on the best method for detecting EGFR mutations. ${ }^{30}$ In Korea, most pathology laboratories use direct DNA sequencing, pyrosequencing, or the peptide nucleic acid (PNA) clamp method for detecting EGFR mutations in formalin-fixed, paraffin-embedded tissue specimens. In the present study, specimens obtained from 14 hospitals were analyzed using direct DNA sequencing, a classic method for detecting mutations. However, this technique is associated with low sensitivity and requires $>25 \%$ mutant DNA for analysis. ${ }^{31}$ Pyrosequencing is a more sensitive method that needs more than $1 \%$ to $20 \%$ mutant DNA for analysis. ${ }^{32,33}$ PNA clamping is a simple, rapid, and sensitive method that can detect mutations in as few as $1 \%$ mutant alleles in a mixture of mutant and wild-type DNA. ${ }^{34}$ These three methods show a good concordance of $82 \%-87.5 \% .{ }^{35,36}$ We recommend that the use of the available methods for EGFR mutation analysis in each institution and laboratory should be under strict quality control. The quality and quantity of DNA are important for avoiding falsenegative results. ${ }^{29}$ In the present study, tumor microdissection specimens from patients with adenocarcinoma showed higher mutation rates than non-dissection specimens. We recommend that pathologists verify the adequacy of specimens and reanalyze EGFR mutations to prevent false-negative results.

According to laboratory status, the EGFR mutation rate in patients with NSCLC (38.2\% vs $23.1 \%, \mathrm{p}<.001)$ and adenocarcinoma ( $44.7 \%$ vs $37.6 \%, \mathrm{p}=.046$ ) was significantly higher in the in-house test in the present study. However, the proportion of never-smoker patients with adenocarcinoma was $48.3 \%$ (628 out 1,299 patients) in the in-house test and 25.1\% (114 out 454 patients) in the out-sourced test. Moreover, EGFR mutation rate in never-smokers with adenocarcinoma was $52.7 \%$ based on inhouse mutation testing and $50.9 \%$ based on out-sourced mutation testing. These results indicate significant differences between in-house and out-sourced mutation testing, which might be because of a bias in patient selection.

In the future, we will aim to develop recommendations for more standardized application and interpretation of results of EGFR mutation tests in patients with NSCLC. These recommendations will discuss patients, turnaround time, specimen type, minimum specimen size, specimen collection and storage, tumor cell content, methodology such as DNA extraction, and reporting form. In addition, we aim to design a QA program for use during EGFR mutation analysis.

The present study had limitations. Pathological diagnosis of patients included in the study was not confirmed. In addition, immunohistochemical staining to classify the histologic type of NSCLC was not performed for all patient samples. Further, our data were collected from hospitals where diagnosis was performed by pulmonary pathologists. Therefore, our results might represent the current status of NSCLC subtypes in Korea.

In conclusion, EGFR mutation rate showed significant differences with respect to sex, smoking history, histologic diagnosis, specimen type, tumor cell dissection, and institution. However, it did not show differences with respect to age, procurement site, or laboratory status. The relative frequency of EGFR mutations in Korea was not similar to those reported in other Asian countries.

\section{Conflicts of Interest}

No potential conflict of interest relevant to this article was reported.

\section{Acknowledgments}

We would like to sincerely thank Seo-Young Oh, MS; NohYoung Lee, MS; and Yoo-Min Hong, MRA for their devoted efforts toward data set mining and cleaning. 


\section{REFERENCES}

1. Jung KW, Won YJ, Kong HJ, et al. Cancer statistics in Korea: incidence, mortality, survival, and prevalence in 2012. Cancer Res Treat 2015; 47: 127-41.

2. Owonikoko TK, Ragin CC, Belani CP, et al. Lung cancer in elderly patients: an analysis of the surveillance, epidemiology, and end results database. J Clin Oncol 2007; 25: 5570-7.

3. Mok TS, Wu YL, Thongprasert S, et al. Gefitinib or carboplatin-paclitaxel in pulmonary adenocarcinoma. N Engl J Med 2009; 361: 947-57.

4. Rosell R, Carcereny E, Gervais R, et al. Erlotinib versus standard chemotherapy as first-line treatment for European patients with advanced EGFR mutation-positive non-small-cell lung cancer (EURTAC): a multicentre, open-label, randomised phase 3 trial. Lancet Oncol 2012; 13: 239-46.

5. Zhou C, Wu YL, Chen G, et al. Erlotinib versus chemotherapy as first-line treatment for patients with advanced EGFR mutationpositive non-small-cell lung cancer (OPTIMAL, CTONG-0802): a multicentre, open-label, randomised, phase 3 study. Lancet Oncol 2011; 12: 735-42.

6. Maemondo M, Inoue A, Kobayashi K, et al. Gefitinib or chemotherapy for non-small-cell lung cancer with mutated EGFR. N Engl J Med 2010; 362: 2380-8.

7. Mitsudomi T, Morita S, Yatabe Y, et al. Gefitinib versus cisplatin plus docetaxel in patients with non-small-cell lung cancer harbouring mutations of the epidermal growth factor receptor (WJTOG3405): an open label, randomised phase 3 trial. Lancet Oncol 2010; 11: 121-8

8. Fukuoka M, Wu YL, Thongprasert S, et al. Biomarker analyses and final overall survival results from a phase III, randomized, open-label, first-line study of gefitinib versus carboplatin/paclitaxel in clinically selected patients with advanced non-small-cell lung cancer in Asia (IPASS). J Clin Oncol 2011; 29: 2866-74.

9. Yang JC, Wu YL, Schuler M, et al. Afatinib versus cisplatin-based chemotherapy for EGFR mutation-positive lung adenocarcinoma (LUX-Lung 3 and LUX-Lung 6): analysis of overall survival data from two randomised, phase 3 trials. Lancet Oncol 2015; 16: 141-51.

10. Yatabe $Y$, Kerr KM, Utomo A, et al. EGFR mutation testing practices within the Asia Pacific region: results of a multicenter diagnostic survey. J Thorac Oncol 2015; 10: 438-45.

11. Yang $\mathrm{CH}$. EGFR tyrosine kinase inhibitors for the treatment of NSCLC in East Asia: present and future. Lung Cancer 2008; 60 Suppl 2: S23-30.

12. Shigematsu H, Lin L, Takahashi T, et al. Clinical and biological features associated with epidermal growth factor receptor gene muta- tions in lung cancers. J Natl Cancer Inst 2005; 97: 339-46.

13. Skov BG, Høgdall E, Clementsen P, et al. The prevalence of EGFR mutations in non-small cell lung cancer in an unselected Caucasian population. APMIS 2015; 123: 108-15.

14. Gahr S, Stoehr R, Geissinger E, et al. EGFR mutational status in a large series of Caucasian European NSCLC patients: data from daily practice. Br J Cancer 2013; 109: 1821-8.

15. Soung YH, Lee JW, Kim SY, et al. Mutational analysis of EGFR and K-RAS genes in lung adenocarcinomas. Virchows Arch 2005; 446: 483-8.

16. Bae NC, Chae MH, Lee MH, et al. EGFR, ERBB2, and KRAS mutations in Korean non-small cell lung cancer patients. Cancer Genet Cytogenet 2007; 173: 107-13.

17. Park S, Holmes-Tisch AJ, Cho EY, et al. Discordance of molecular biomarkers associated with epidermal growth factor receptor pathway between primary tumors and lymph node metastasis in nonsmall cell lung cancer. J Thorac Oncol 2009; 4: 809-15.

18. Lee YJ, Park IK, Park MS, et al. Activating mutations within the EGFR kinase domain: a molecular predictor of disease-free survival in resected pulmonary adenocarcinoma. J Cancer Res Clin Oncol 2009; 135: 1647-54.

19. Ahn MJ, Park BB, Ahn JS, et al. Are there any ethnic differences in molecular predictors of erlotinib efficacy in advanced non-small cell lung cancer? Clin Cancer Res 2008; 14: 3860-6.

20. Sun PL, Seol H, Lee HJ, et al. High incidence of EGFR mutations in Korean men smokers with no intratumoral heterogeneity of lung adenocarcinomas: correlation with histologic subtypes, EGFR/ TTF-1 expressions, and clinical features. J Thorac Oncol 2012; 7: 323-30.

21. Kim YT, Seong YW, Jung YJ, et al. The presence of mutations in epidermal growth factor receptor gene is not a prognostic factor for long-term outcome after surgical resection of non-small-cell lung cancer. J Thorac Oncol 2013; 8: 171-8.

22. Choi YL, Sun JM, Cho J, et al. EGFR mutation testing in patients with advanced non-small cell lung cancer: a comprehensive evaluation of real-world practice in an East Asian tertiary hospital. PLoS One 2013; 8: e56011.

23. Ma BB, Hui EP, Mok TS. Population-based differences in treatment outcome following anticancer drug therapies. Lancet Oncol 2010; 11: 75-84.

24. Chan SK, Gullick WJ, Hill ME. Mutations of the epidermal growth factor receptor in non-small cell lung cancer: search and destroy. Eur J Cancer 2006; 42: 17-23.

25. Shi Y, Au JS, Thongprasert S, et al. A prospective, molecular epidemiology study of EGFR mutations in Asian patients with advanced non-small-cell lung cancer of adenocarcinoma histology (PIO- 
NEER). J Thorac Oncol 2014; 9: 154-62.

26. Liu J, Zhao R, Zhang J, Zhang J. ARMS for EGFR mutation analysis of cytologic and corresponding lung adenocarcinoma histologic specimens. J Cancer Res Clin Oncol 2015; 141: 221-7.

27. Heymann JJ, Bulman WA, Maxfield RA, et al. Molecular testing guidelines for lung adenocarcinoma: utility of cell blocks and concordance between fine-needle aspiration cytology and histology samples. Cytojournal 2014; 11: 12.

28. Khode R, Larsen DA, Culbreath BC, et al. Comparative study of epidermal growth factor receptor mutation analysis on cytology smears and surgical pathology specimens from primary and metastatic lung carcinomas. Cancer Cytopathol 2013; 121: 361-9.

29. Sun PL, Jin Y, Kim H, Lee CT, Jheon S, Chung JH. High concordance of EGFR mutation status between histologic and corresponding cytologic specimens of lung adenocarcinomas. Cancer Cytopathol 2013; 121: 311-9.

30. Pirker R, Herth FJ, Kerr KM, et al. Consensus for EGFR mutation testing in non-small cell lung cancer: results from a European workshop. J Thorac Oncol 2010; 5: 1706-13.

31. Pao W, Ladanyi M. Epidermal growth factor receptor mutation testing in lung cancer: searching for the ideal method. Clin Cancer
Res 2007; 13: 4954-5.

32. Kim SK, Kim DL, Han HS, et al. Pyrosequencing analysis for detection of a BRAFV600E mutation in an FNAB specimen of thyroid nodules. Diagn Mol Pathol 2008; 17: 118-25.

33. Dufort S, Richard MJ, Lantuejoul S, de Fraipont F. Pyrosequencing, a method approved to detect the two major EGFR mutations for anti EGFR therapy in NSCLC. J Exp Clin Cancer Res 2011; 30: 57.

34. Choi JJ, Cho M, Oh M, Kim H, Kil MS, Park H. PNA-mediated real-time PCR clamping for detection of EGFR mutations. Bull Korean Chem Soc 2010; 31: 3525-9.

35. Lee HJ, Xu X, Kim H, et al. Comparison of direct sequencing, PNA clamping-real time polymerase chain reaction, and pyrosequencing methods for the detection of EGFR mutations in non-small cell lung carcinoma and the correlation with clinical responses to EGFR tyrosine kinase inhibitor treatment. Korean J Pathol 2013; 47: 52-60.

36. Kim HJ, Lee KY, Kim YC, et al. Detection and comparison of peptide nucleic acid-mediated real-time polymerase chain reaction clamping and direct gene sequencing for epidermal growth factor receptor mutations in patients with non-small cell lung cancer. Lung Cancer 2012; 75: 321-5. 